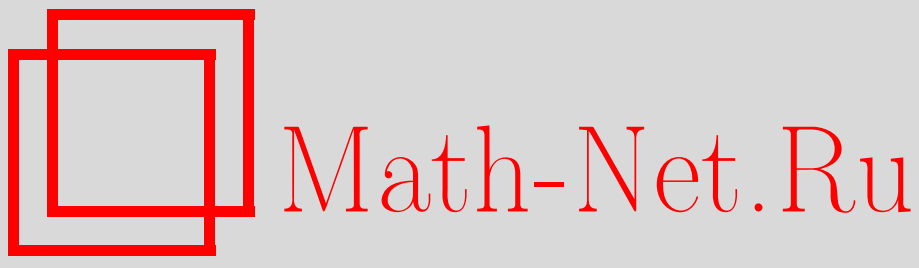

Л. И. Камынин, Б. Н. Химченко, Об априорных оценках решения І-ой краевой задачи для одного класса параболических систем второго порядка, Изв. РАН. Сер. матем., 2001, том 65, выпуск 4, 67-88

DOI: https://doi.org/10.4213/im348

Использование Общероссийского математического портала Math-Net.Ru подразумевает, что вы прочитали и согласны с пользовательским соглашением

http://www.mathnet.ru/rus/agreement

Параметры загрузки:

IP: 107.22 .136 .117

26 апреля 2023 г., 18:12:08 
УДК 517.9

\author{
Л. И. Камынин, Б.Н. Химченко
}

\title{
Об априорных оценках решения I-ой краевой задачи для одного класса параболических систем второго поря,дка
}

\begin{abstract}
Рассмотрены два класса параболических матрично-векторных систем второго порядка (с решением $u \in M_{m \times 1}, m \geqslant 2$ ), допускающих сведение их к одному скалярному параболическому уравнению второго порядка для числовой функции $v=\langle p, u\rangle$, где $p \in M_{m \times 1}$ - фиксированный стохастический постоянный вектор. В неограниченной (по $x$ ) области рассмотрена I-я краевая задача для скалярного параболического уравнения второго порядка (с неограниченными коэффициентами), удовлетворяющая условию сильного поглощения на бесконечности. В обобщенных классах Тихонова-Тэклинда, обеспечивающих единственность решения, доказана априорная оценка решения I-ой краевой задачи.

Библиографиия: 11 наименований.
\end{abstract}

\section{Введение}

В работах [1], [2] при рассмотрении вопроса о слабом принципе экстремума для параболических систем второго порядка был изложен метод сведения матричновекторной параболической системы к одному скалярному параболическому уравнению второго порядка

$$
L v=-H
$$

для числовой функции $v(x, t)=\langle p, u(x, t)\rangle$, где $u \in M_{m \times 1}-$ решение параболической системы, а $p$ - стохастический вектор.

Для полученного параболического уравнения второго порядка (0.1) справедлив классический слабый принцип экстремума (см., например, [2], [6]-[9]), который можно трактовать как слабый принцип экстремума (СПЭ) для исходной параболической системы. Введенный таким образом СПЭ используется в статье для нахождения априорных оценок классического решения I-ой краевой задачи для параболической системы (точнее, для скалярного уравнения (0.1), соответствуюшего исходной системе) в неограниченной (по $x$ ) области в обобщенных классах быстро растущих (по $x$ ) функций Тихонова-Тэклинда (ОТТ), введенных в [3], [4] и обеспечивающих, в частности, единственность решения І-ой краевой задачи. Основное внимание обращено при этом на априорные оценки для уравнения $(0.1)$ с условием сильного поглошения на бесконечности.

Статья состоит из двух параграфов.

В $§ 1$ приведены найденные в [1], [2] классы параболических систем второго порядка (с полным перемешиванием), допускаюших сведение к одному скаляр-

(С) Л.И. КАмынин, Б.Н. Химченко, 2001 
ному параболическому уравнению второго порядка (0.1), для которого справедлив СПЭ, который и трактуется как СПЭ для исходной системы. Основным моментом работы [1] (см. [1, условие (А.1), с. 47]) является возможность разложения матрищ-коэффищиентов исходной матрично-векторной системы как в матрицы простой структуры (подобные диагональным), так и в нильпотентные матрицы (см. [5]). Основным моментом работы [2] является использование условия (Р) о существовании стохастической матрицы $P$ (см. [2, с. 60]), после умножения на которую слева исходная параболическая система второго порядка приводится к скалярному параболическому уравнению (0.1). В $\S 1$ сформулирован СПЭ для уравнения (0.1) и приведены как необходимые (теорема 1.1), так и достаточные (теоремы 1.2 и 1.3) условия его справедливости.

В $\S 2$ устанавливается априорная оценка решения I-ой краевой задачи для уравнения (0.1) в неограниченной (по $x$ ) области $\omega \subset \mathbb{R}_{x, t}^{n+1}$ в классах ОТТ, обеспечивающих единственность решения краевой задачи. Точный класс единственности ОТТ соответствует при этом заданному поведению (при $|x| \rightarrow+\infty)$ наибольшего собственного значения $\lambda_{n}(x, t)$ характеристической формы параболического оператора второго порядка $L v$ из (0.1) в трех вариантах:

1) $\lim _{|x| \rightarrow+\infty} \lambda_{n}(x, t)=+\infty$;

2) ограниченность $\lambda_{n}(x, t)$ в $\omega$;

3) $\lim _{|x| \rightarrow+\infty} \lambda_{n}(x, t)=0$.

Коэффищиенты оператора $L$ при $D_{i} v$ могут при этом расти (по модулю) к бесконечности, но не быстрее функции Тэклинда (см. ниже определение 2.2). На априорных оценках решения I-ой краевой задачи для уравнения (0.1) существенно сказывается и поведение коэффициента $c(x, t)$ (при $v$ в операторе $L v$ ), для чего выделены три варианта поведения при $|x| \rightarrow+\infty$ :

1 ) ограниченность $c(x, t)$ (что соответствует слабой диссипативности $L v$ при $-C \leqslant c(x, t) \leqslant 0$, слабого поглошения $L v$ при $0 \leqslant c(x, t) \leqslant C)$;

2) $\lim _{|x| \rightarrow+\infty} c(x, t)=-\infty$ (сильная диссипативность $\left.L v\right)$;

3) $\lim _{|x| \rightarrow+\infty} c(x, t)=+\infty$ (условие сильного поглошения для $\left.L v\right)$.

Априорные оценки решения I-ой краевой задачи для уравнения (0.1) рассматривались (при ограниченных коэффищиентах $L v$ ) в [6]-[9]. В случае выполнения условия сильной диссипативности 2) априорные оценки решения І-ой краевой задачи были получены в [11]. В $§ 2$ методом барьерных функций устанавливаются априорные оценки решения І-ой краевой задачи для уравнения $(0.1)$ в неограниченных (по $x$ ) областях в классе ОТТ при выполнении условия сильного поглощения 3), т.е. при наличии внутренних источников или сильного поглощения из внешней среды.

\section{§1. О слабом принципе экстремума для двух классов параболических систем второго порядка}

Пусть $\omega \subset\{0<t<T<+\infty\} \subset \mathbb{R}_{x, t}^{n+1}, n \geqslant 1,-$ область, для которой

$$
\partial \omega=\gamma_{0}(\omega) \cup \Sigma(\omega) \cup \gamma(\omega)
$$


где нижняя крышка $\gamma_{0}(\omega)=\operatorname{int}(\bar{\omega} \cap\{t=0\}) \subset \mathbb{R}_{x}^{n}$ является областью на гиперплоскости $\{t=0\}$, а верхняя крьшка $\gamma(\omega)=\operatorname{int}(\bar{\omega} \cap\{t=T>0\})$ является областью на гиперплоскости $\{t=T>0\}$ и

$$
\Gamma(\omega)=\partial \omega \backslash \gamma(\omega) \equiv \gamma_{0}(\omega) \cup \Sigma(\omega)
$$

- параболическая гранища области $\omega$ с боковой частью

$$
\Sigma(\omega)=\Gamma(\omega) \backslash \gamma_{0}(\omega)
$$

Рассмотрим на множестве $\omega \cup \gamma(\omega) \subset \mathbb{R}_{x, t}^{n+1}$ параболическую систему второго порядка из $m(m \geqslant 2)$ уравнений с "полным перемешиванием" вида

$$
\begin{aligned}
L_{r} u & \equiv \sum_{k=1}^{m}\left(\sum_{i, j=1}^{n} a_{k, i j}^{(r)}(M) D_{i} D_{j} u_{k}+\sum_{i=1}^{n} b_{k, i}^{(r)}(M) D_{i} u_{k}+c_{k}^{(r)}(M) u_{k}\right)-D_{t} u_{k} \\
& =-\Phi(M), \quad r=\overline{1, m}
\end{aligned}
$$

где $D_{i}=\frac{\partial}{\partial x_{i}}, i=\overline{1, n}, D_{t}=\frac{\partial}{\partial t}$ и при $M=(x, t) \equiv\left(x_{1}, \ldots, x_{n} ; t\right), n \geqslant 1$, действительные функции $a_{k, i j}^{(r)}, b_{k, i}^{(r)}, c_{k}^{(r)}$ принадлежат $C(\omega \cup \gamma(\omega)), r, k=\overline{1, m}$, $i, j=\overline{1, n}$, причем матрицы

$$
a_{k}^{(r)}(M)=\left[a_{k, i j}^{(r)}(M)\right] \in M_{n \times n}, \quad r, k=\overline{1, m},
$$

предполагаются симметрическими и положительно определенньми на множестве $\omega \cup \gamma(\omega)$, так что их собственные значения

$$
0<\lambda_{k, 1}^{(r)}(M) \leqslant \cdots \leqslant \lambda_{k, n}^{(r)}(M)<+\infty \quad \forall M \in \omega \cup \gamma(\omega)
$$

положительны.

Введем матрицы

$$
\begin{gathered}
A_{i j}=\left[a_{k, i j}^{(r)}\right], \quad B_{i}=\left[b_{k, i}^{(r)}\right], \quad C(M)=\left[c_{k}^{(r)}\right], \\
I_{m}=\left[\begin{array}{lll}
1 & & 0 \\
& \ddots & \\
& & \\
0 & & 1
\end{array}\right]=\left[e_{1} \ldots e_{m}\right] \in M_{m \times m}, \quad i, j=\overline{1, n}
\end{gathered}
$$

и векторы

$$
\begin{gathered}
u=\left[u_{1} \ldots u_{m}\right]^{T}, \quad D_{i} u=\left[D_{i} u_{1} \ldots D_{i} u_{m}\right]^{T}, \\
D_{i} D_{j} u=\left[D_{i} D_{j} u_{1} \ldots D_{i} D_{j} u_{m}\right]^{T}, \quad D_{t} u=I_{m} D_{t} u=\left[D_{t} u_{1} \ldots D_{t} u_{m}\right]^{T}, \\
\Phi=\left[\Phi_{1} \ldots \Phi_{m}\right]^{T},
\end{gathered}
$$

принадлежашие $M_{m \times 1}$. 
Тогда параболическую систему второго порядка (1.1) можно записать в матрично-векторном виде:

$$
\begin{aligned}
L u & \equiv \sum_{i, j=1}^{n} A_{i j}(M) D_{i} D_{j} u+\sum_{i=1}^{n} B_{i}(M) D_{i} u+C(M) u-I_{m} D_{t} u \\
& =-\Phi(M) \quad \forall M \in \omega \cup \gamma(\omega) .
\end{aligned}
$$

Напомним, что в [1] было введено условие (А.1) (см. [1, определение 1 при $k=1$, c. 47]) для матрищ (1.3), позволяющее свести матрично-векторную параболическую систему (1.4), имеюшую решение

$$
u \in C_{x, t}^{(2,1)}(\omega \cup \gamma(\omega)), \quad u \in M_{m \times 1},
$$

к одному скалярному уравнению второго порядка

$$
\begin{aligned}
L v \equiv & \frac{1}{s_{1}} \sum_{i, j=1}^{n}\left(\sum_{r=1}^{m} s_{r} a_{1, i j}^{(r)}(M)\right) D_{i} D_{j} v+\frac{1}{s_{1}} \sum_{i=1}^{n}\left(\sum_{r=1}^{m} s_{r} b_{1, i}^{(r)}(M)\right) D_{i} v \\
& +\frac{1}{s_{1}}\left(\sum_{r=1}^{m} s_{r} c_{1}^{(r)}(M)\right) v-D_{t} v \equiv-H(M) \equiv-\langle p, \Phi(M)\rangle
\end{aligned}
$$

где

$$
v(M)=\langle p, u(M)\rangle \in C_{x, t}^{(2,1)}(\omega \cup \gamma(\omega))
$$

при положительном стохастическом (см. [5]) векторе

$$
p=\left[s_{1} \ldots s_{m}\right]^{T} \in M_{m \times 1}, \quad s_{r}>0 \quad \forall r=\overline{1, m}, \quad \sum_{k=1}^{m} s_{k}=1 .
$$

В силу стохастичности вектора (1.7) имеем оценку

$$
\frac{1}{s_{1}} \sum_{i, j=1}^{n}\left(\sum_{r=1}^{m} s_{r} a_{1, i j}^{(r)}(M)\right) \xi_{i} \xi_{j}>0 \quad \forall M \in \omega \cup \gamma(\omega), \quad|\xi|_{n}=1,
$$

показывающую параболичность уравнения (1.5), причем условие диссипативности (1.5) имеет вид

$$
\frac{1}{s_{1}} \sum_{r=1}^{m} s_{r} c_{1}^{(r)}(M) \leqslant 0 \quad \forall M \in \omega \cup \gamma(\omega) .
$$

В работе [2] была введена стохастическая матрица (см. (1.7))

$$
P=\left[\begin{array}{ccc}
s_{1} & \ldots & s_{m} \\
\ldots & \ldots & \ldots \\
s_{1} & \ldots & s_{m}
\end{array}\right] \in M_{m \times m}
$$

ОПРЕДЕЛЕНИЕ 1.1 (см. [2, с. 60]). Матрица $A \in M_{m \times m}$ удовлетворяет условию $(\mathrm{P})$, если для стохастической матрищы (1.8) выполнено равенство

$$
P A=P A P \text {. }
$$


ЗАмечание 1.1 (критерий выполнения условия (Р)). Матрища $A=\left[A_{i j}\right] \in$ $M_{m \times m}$ удовлетворяет условию $(\mathrm{P})$ тогда и только тогда, когда

$$
\sum_{k=1}^{m} s_{k} A_{k r}=s_{r} \sum_{i, j=1}^{m} s_{i} A_{i j} \quad \forall r=\overline{1, m} .
$$

ОПРЕДЕЛЕнИЕ 1.2. Параболическая система второго порядка (1.4) удовлетворяет условию $(\mathrm{P})$, если существует стохастический вектор (1.7), для которого каждая матрица из (1.3) удовлетворяет условию $(\mathrm{P})$ определения 1.1 в общей стохастической матрище (1.8) такой, что $\forall M=(x, t) \in \omega \cup \gamma(\omega)$ имеют место равенства

$$
\begin{gathered}
P A_{i j}(M)=P A_{i j}(M) P, \quad P B_{i}(M)=P B_{i}(M) P \quad \forall i, j=\overline{1, n} \\
P C(M)=P C(M) P, \quad P I_{m}=P I_{m} P .
\end{gathered}
$$

В [2] показано, что после умножения системы (1.4) слева на (1.8) и использования (1.9) числовая функция (1.6) становится решением на множестве $\omega \cup \gamma(\omega)$ скалярного уравнения второго порядка

$$
\begin{aligned}
L v & \equiv \sum_{i, j=1}^{n} a_{i j}(M) D_{i} D_{j} v+\sum_{i=1}^{n} b_{i}(M) D_{i} v+c(M) v-D_{t} v \\
& =-H(M) \equiv-\langle p, \Phi(M)\rangle
\end{aligned}
$$

где положено

$$
\begin{gathered}
a_{i j}(M)=\sum_{r, k=1}^{m} s_{r} a_{k, i j}^{(r)}(M), \quad b_{i}(M)=\sum_{r, k=1}^{m} s_{r} b_{k, i}^{(r)}(M), \quad i, j=\overline{1, n} \\
c(M)=\sum_{r, k=1}^{m} s_{r} c_{k}^{(r)}(M), \quad H(M)=-\langle p, \Phi(M)\rangle
\end{gathered}
$$

причем матрица $\left[a_{i j}(M)\right] \in M_{n \times n}$ из (1.10) симметрическая и в силу стохастичности вектора (1.7) положительно определенная, так что уравнение второго порядка (1.10) параболично на $\omega \cup \gamma(\omega)$ с условием диссипативности

$$
c(M) \leqslant 0 \quad \forall M \in \omega \cup \gamma(\omega) .
$$

Итак, при выполнении условий (А.1) из [1] или (Р) из [2] параболическая система второго порядка (1.1), (1.4) может быть сведена к одному (скалярному) уравнению второго порядка параболического типа для числовой функции (1.6) вида (1.10), причем

$$
a_{i j}(M)=\left\{\begin{array}{cc}
\frac{1}{s_{1}} \sum_{r=1}^{m} s_{r} a_{1, i j}^{(r)}(M) & \text { при (А.1), } \\
\sum_{r, k=1}^{m} s_{r} a_{k, i j}^{(r)}(M) & \text { при }(\mathrm{P}),
\end{array}\right.
$$




$$
\begin{aligned}
b_{i}(M) & = \begin{cases}\frac{1}{s_{1}} \sum_{r=1}^{m} s_{r} b_{1, i}^{(r)}(M) & \text { при (А.1), } \\
\sum_{r, k=1}^{m} s_{r} b_{k, i}^{(r)}(M) & \text { при }(\mathrm{P}),\end{cases} \\
c(M) & = \begin{cases}\frac{1}{s_{1}} \sum_{r=1}^{m} s_{r} c_{1}^{(r)}(M) & \text { при (А.1), } \\
\sum_{r, k=1}^{m} s_{r} c_{k}^{(r)}(M) & \text { при }(\mathrm{P}) .\end{cases}
\end{aligned}
$$

ОПРЕДЕЛЕНИЕ 1.3 (cp. [2], [6]-[9]). Для параболического уравнения второго порядка (1.10), заданного на ограниченном множестве $\omega \cup \gamma(\omega) \subset \mathbb{R}_{x, t}^{n+1}, n \geqslant 1$, справедлив слабый принцип минимума (СПМин), соответственно слабый принцип максимума (СПМах), если для любого супер(суб)параболического решения (1.6) уравнения (1.10), т.е. при $-H(M) \leqslant 0$ или $-H(M)<0$ (соответственно $-H(M) \geqslant 0$ или $-H(M)>0) \forall M=(x, t) \in \omega \cup \gamma(\omega)$ не существует точки $M_{0}=\left(x_{0}, t_{0}\right) \in \omega \cup \gamma(\omega)$ и полуокрестности

$$
U^{-}\left(M_{0}\right)=\left\{(x, t)|| x-x_{0} \mid<\rho, t_{0}-\rho<t \leqslant t_{0}\right\} \subset \omega \cup \gamma(\omega)
$$

таких, что выполнена оценка снизу

$$
v(M) \geqslant v\left(M_{0}\right) \quad \forall M \in U^{-}\left(M_{0}\right),
$$

где либо $v\left(M_{0}\right)<0$ при $-H\left(M_{0}\right) \leqslant 0$, либо $v\left(M_{0}\right) \leqslant 0$ при $-H\left(M_{0}\right)<0$ (соответственно выполнена оценка сверху

$$
v(M) \leqslant v\left(M_{0}\right) \quad \forall M \in U^{-}\left(M_{0}\right),
$$

где либо $v\left(M_{0}\right)>0$ при $-H\left(M_{0}\right) \geqslant 0$, либо $v\left(M_{0}\right) \geqslant 0$ при $\left.-H\left(M_{0}\right)>0\right)$.

ОПРЕДЕЛЕниЕ 1.4 (ср. [1], [2]). Для параболической системы второго порядка (1.1), (1.4), заданной на ограниченном множестве $\omega \cup \gamma(\omega)$ и имеющей решение $u \in M_{m \times 1}$ (см. (1.3)) класса $C_{x, t}^{(2,1)}(\omega \cup \gamma(\omega))$, справедлив СПМин (соответственно СПМах), если выполнены условия:

1) параболическая система второго порядка (1.4) удовлетворяет или условию (А.1) из [1], или условию (Р) из [2] при фиксированном стохастическом вектоpe (1.7);

2) для скалярного параболического уравнения второго порядка (1.10), (1.11), соответствуюшего системе (1.4) при $v(M)$ из (1.6) и $H(M)=\langle p, \Phi(M)\rangle$, справедлив СПМин (СПМах) в смысле определения 1.3. 
TЕоРема 1.1 (о необходимом условии выполнения СПМин для (1.10) [2]). Пусть оператор Lv из (1.10) имеет непрерывные коэффициенты (1.11) на ограниченном мнохсестве $\omega \cup \gamma(\omega)$, причем

$$
c(M) \neq 0, \quad \alpha(M) \equiv \sum_{i=1}^{n} a_{i i}(M)>0 \quad \forall M \in \omega \cup \gamma(\omega)
$$

и существует точка $M_{0}=\left(x_{0}, t_{0}\right) \in \omega \cup \gamma(\omega)$, в которой не выполнено условие “диссипативности" для уравнения (1.10) (см. (1.12)):

$$
c(M)<0 \quad \forall M \in \omega \cup \gamma(\omega),
$$

mak чmo

$$
c\left(M_{0}\right)=\frac{4}{\gamma_{0}}>0 .
$$

Тогда:

1) существуют полуокрестность $U^{-}\left(M_{0}\right) \subset \omega \cup \gamma(\omega)$ и отричательная непрерывная в $U^{-}\left(M_{0}\right)$ функция $f(M)<0, f\left(M_{0}\right)=-1$, такие, что

2) функиия (см. (1.13))

$$
v(x, t)=-\gamma_{0}+t_{0}-t+\frac{\left|x-x_{0}\right|^{2}}{\alpha\left(M_{0}\right)}
$$

класса $C_{x, t}^{(2,1)}\left(U^{-}\left(M_{0}\right)\right)$ удовлетворяет уравнению (см. (1.10))

$$
L v(M)=f(M)<0 \quad \forall M \in U^{-}\left(M_{0}\right)
$$

с правой частью $f(M)$ из утверждения 1$)$, причем

$$
L v\left(M_{0}\right)=-1 \equiv f\left(M_{0}\right)
$$

(т.е. $v(M)$ суперпараболична в полуокрестности $\left.U^{-}\left(M_{0}\right)\right)$ u

$$
v(M) \geqslant v\left(M_{0}\right)=-\gamma_{0} \quad \forall M \in U^{-}\left(M_{0}\right),
$$

где постоянная $\gamma_{0}>0$ взята из (1.13) (так что для уравнения (1.10) в $U^{-}\left(M_{0}\right)$ не выполнен классический СПМин).

Теорема 1.2 (достаточное условие вьполнения СПМин для (1.10) [2]). Пусть для параболического уравнения второго порядка (1.10), заданного на ограниченном множестве $\omega \cup \gamma(\omega), \quad \forall M \in \omega \cup \gamma(\omega)$ выполнено одно из условий: либо $-H(M)<0$ и с $(M) \leqslant 0$, либо $-H(M) \leqslant 0$, но $c(M)<0$. Тогда для параболического уравнения (1.10), (1.11) справедлив СПМин на $\omega \cup \gamma(\omega)$ в смысле определения 1.3. 
ТЕорема 1.3 (об усиленном СПМин (СПМах) для параболической системы второго порядка (1.4)). Пусть на ограниченном множестве $\omega \cup \gamma(\omega) \subset \mathbb{R}_{x, t}^{n+1}$ задана параболическая система второго порядка (1.1), (1.4) при положительных значениях (1.2) матриц

$$
\left[a_{k, i j}^{(r)}(M)\right] \in M_{n \times n}, \quad r, k=\overline{1, m},
$$

из (1.3), причем непрерывные на $\omega \cup \gamma(\omega)$ матричы (1.3) удовлетворяют либо условию (А.1) из [1], либо условию (P) из [2] при выполнении ослабленного условия диссипативности

$$
c(M) \leqslant 0 \quad \forall M \in \omega \cup \gamma(\omega)
$$

для уравнения (1.10), (1.11). Тогда для решения $v(M)$ из (1.6) скалярного уравнения второго порядка (1.10), (1.11), соответствующего системе (1.4), имеем

$$
\min _{\bar{\omega}} v(M)=\min _{\Gamma(\omega)} v \quad n p u \quad \min _{\bar{\omega}} v \leqslant 0
$$

соответственно

$$
\max _{\bar{\omega}} v(M)=\max _{\Gamma(\omega)} v \quad n p u \quad \max _{\bar{\omega}} v \geqslant 0
$$

и, в частности, в силу стохастичности вектора (1.7)

$$
\left.u\right|_{\Gamma(\omega)} \geqslant 0 \Rightarrow v(M) \equiv\langle p, u(M)\rangle \geqslant 0 \quad \forall M \in \bar{\omega}
$$

соответственно

$$
\left.u\right|_{\Gamma(\omega)} \leqslant 0 \Rightarrow v(M) \leqslant 0 \quad \forall M \in \bar{\omega} .
$$

ДоКАЗАТЕЛЬСТВО сводится к доказательству усиленного слабого принщипа экстремума для решения $v(M)$ из (1.6) уравнения (1.10), (1.11) и проводится по схеме соответствуюшего утверждения для уравнения теплопроводности из [9] (см., например, доказательство теоремы 6 из [2]).

\section{§2. Априорные оценки решения І-ой краевой задачи}

в неограниченной (по $x$ ) области для параболического уравнения второго порядка (1.10) с условием сильного поглощения

В этом параграфе будем рассматривать неограниченную (по $x$ ) область $\omega \subset$ $\Pi(0,+\infty)$, где

$$
\Pi(0,+\infty)=\left\{(x, t) \in \mathbb{R}_{x, t}^{n+1}|| x \mid<+\infty, 0<t<+\infty\right\},
$$

с границей (ср. §1) $\partial \omega=\gamma_{0}(\omega) \cup \Sigma(\omega)$. 
Положим также

$$
\Pi(0, T]=\Pi(0,+\infty) \cap\{0<t \leqslant T<+\infty\}
$$

и для каждого $T>0$ определим множество $\omega_{T}=\omega \cap \Pi(0, T]$ такое, что $\gamma_{0}\left(\omega_{T}\right)=$ $\gamma_{0}(\omega)$ и верхняя крьшка

$$
\gamma\left(\omega_{T}\right)=\operatorname{int}(\bar{\omega} \cap\{t=T>0\}) \subset \mathbb{R}_{x}^{n}
$$

является областью на гиперплоскости $\{t=T>0\}$, причем

$$
\Sigma\left(\omega_{T}\right)=\partial \omega_{T} \backslash\left(\gamma_{0}(\omega) \cup \gamma\left(\omega_{T}\right)\right)
$$

Введем также параболическую границу множества $\omega_{T}$

$$
\Gamma\left(\omega_{T}\right)=\Sigma\left(\omega_{T}\right) \cup \gamma_{0}(\omega) \quad \forall T>0
$$

ОПРЕДЕлЕНИЕ 2.1. Пусть для области $\omega \subset \Pi(0,+\infty)$ существует точка $(y, 0)$ такая, что $\{|x-y|<\rho, 0 \leqslant t<+\infty\} \subset C \bar{\omega}$, причем, без ограничения обшности, будем считать в дальнейшем, что $y=0$, так что $\forall(x, t) \in \omega$ имеем $|x|>\rho>0$.

ОПРЕДЕЛЕНИЕ 2.2. Числовая функция $h \in C^{(1)}[0,+\infty)$ называется функцией Тәклинда, если:

1) $h(s)=h_{0}=h(1) \forall s \in[0,1], h(s) \geqslant h_{0} s \forall s \geqslant s_{0}>1$, причем

$$
\lim _{s \rightarrow+\infty} \frac{s}{h(s)}=0
$$

2) $D h(s) \geqslant 0 \forall s \in\left[0, s_{0}\right], D h(s)>0 \forall s \geqslant s_{0}>1$,

$$
\max _{s \in\left[1, s_{0}\right]} \operatorname{Dh}(s)=d_{0}>0
$$

3) (условие Тэклинда)

$$
\int_{0}^{+\infty} \frac{d s}{h(s)}=+\infty
$$

ПрИМЕР. Имеем

$$
h(s)=s \prod_{k=1}^{m} \ln _{k} s \quad \forall s \geqslant s_{0}=\exp _{m} e
$$

где

$$
\ln _{k} s=\underbrace{\ln \ldots \ln }_{k} s, \quad \exp _{k} s=\underbrace{\exp \ldots \exp }_{k} s .
$$


ЗАмечанИЕ 2.1. Аддитивным классом $T(h)$ называется совокупность функций Тэклинда такая, что для любого конечного числа $l \geqslant 2$ функций Тэклинда $h_{k} \in T(h), k=\overline{1, l}$, функция $\sum_{k=1}^{l} h_{k}$ также является функцией Тэклинда (примеры см. в [3]).

ОПРЕДЕЛЕниЕ 2.3. Классом $T_{h}(g)$ называется совокупность функций $g \in$ $C^{(1)}[0,+\infty)$, удовлетворяющих условиям:

1) $g(s)=g_{0}>0 \forall s \in\left[0, s_{0}\right], g(s)>0 \forall s \geqslant 0$;

2) $\int_{0}^{+\infty} \frac{d s}{g(s)}=+\infty$, так что для функции $G(s)=\int_{0}^{s} \frac{d z}{g(z)}$ имеем

$$
G(s)=\frac{s}{g_{0}} \forall s \in\left[0, s_{0}\right], \quad D G(s)=\frac{1}{g(s)}>0 \quad \forall s>0
$$

и $\lim _{s \rightarrow+\infty} G(s)=+\infty$;

3) $-D g(s) \leqslant h(G(s)) \forall s \geqslant s_{0}>0$, где $h \in T(h)$;

4) $0<D h(s) \leqslant q h^{2}(s) \forall s \geqslant s_{0}>1, q>0$.

ЗАмЕчАниЕ 2.2. Для функции $g \in T_{h}(g)$ возможны следующие варианты поведения при $s \rightarrow+\infty$ :

1) $\lim _{s \rightarrow+\infty} g_{1}(s)=+\infty, g_{1}(s)=s \prod_{k=1}^{m} \ln _{k} s \forall s \geqslant s_{0}$;

2) $0<g_{2}(s) \leqslant A<+\infty \forall s \geqslant 0, g_{2}(s) \equiv 1$;

3) $\lim _{s \rightarrow+\infty} g_{3}(s)=0, g_{3}(s)=s^{-\alpha} \forall s \geqslant s_{0}, \alpha<0$.

В этом параграфе будем считать, что параболическая система второго порядка (1.4), рассматриваемая в неограниченной (по $x$ ) области $\omega$, удовлетворяюшей определению 2.1 , сводится (см. $§ 1$ ) к скалярному параболическому уравнению второго порядка $(1.10),(1.11)$. Тогда из (1.2) для наибольшего собственного значения $\lambda_{n}(x, t)$ характеристической формы уравнения (1.10), (1.11) получаем

$$
\lambda_{n}(x, t)=\left\{\begin{array}{cc}
\frac{1}{s_{1}} \sum_{r=1}^{m} s_{r} \lambda_{1, n}^{(r)}(x, t) & \text { при (А.1), } \\
\sum_{r, k=1}^{m} s_{r} \lambda_{k, n}^{(r)}(x, t) & \text { при (Р). }
\end{array}\right.
$$

Пусть $h \in T(h), g \in T_{h}(g)$ и функция (1.6) из класса $C(\bar{\omega}) \wedge C_{x, t}^{(2,1)}(\omega)$ взята из обобщенного класса Тихонова-Тэклинда (ОТТ), введенного в [3], [4] так, что (см. определения $2.2,2.3$ )

$$
|v(x, t)| \leqslant v_{0}(t) \exp \left\{\int_{0}^{G(|x|)} h(s) d s\right\} \quad \forall(x, t) \in \bar{\omega},
$$

где функция $G$ взята из условия 2$)$ определения 2.3 и $v_{0}(t)$ - положительная, неубывающая, непрерывная числовая функция на $[0,+\infty)$.

Скажем, что функция $v(M)$ из (1.6) является решением I-ой краевой задачи для уравнения (1.10), если

$$
L v(x, t)=-H(x, t) \quad \forall(x, t) \in \omega
$$


при начальном условии

$$
v(x, 0)=f(x) \quad \forall(x, 0) \in \bar{\gamma}_{0}(\omega)
$$

и краевом условии 1-го рода

$$
v\left(M_{0}\right)=\varphi\left(M_{0}\right) \quad \forall M_{0}=\left(x_{0}, t_{0}\right) \in \Sigma_{0}(\omega)=\Sigma(\omega) \backslash \bar{\gamma}_{0}(\omega) .
$$

ЗАМЕЧАНИЕ 2.3 (см. определения 2.1-2.3). Положим при $h, h_{c} \in T(h), g \in$ $T_{h}(g)$

$$
\begin{gathered}
G_{0}=\frac{s_{0}}{g_{0}} \geqslant G(s)=\frac{s}{g_{0}} \quad \forall s \in\left[0, s_{0}\right], \\
g(s)=g_{0}>0 \forall s \in\left[0, s_{0}\right], \quad h(s)= \begin{cases}h_{0}, & 0 \leqslant s \leqslant 1, \\
\geqslant h_{0} s, & 1<s \leqslant s_{0}\end{cases} \\
D h(s)= \begin{cases}0, & s \in[0,1], \\
0<D h(s) \leqslant d_{0} & \forall s \in\left[1, s_{0}\right] .\end{cases}
\end{gathered}
$$

Пусть $0<r<1<4<R<+\infty, r X>s_{0}>0, \alpha=\frac{R}{R-1}>0$ и $\rho>0$;

$$
\begin{aligned}
& A=\frac{\alpha h^{2}\left(G_{0}\right)+d_{0}}{2(3+q)}+\frac{g_{0}}{8} h^{2}\left(G_{0}\right), \\
& B=\frac{\alpha h^{2}\left(G_{0}\right)+d_{0}}{2(3+q)}+\frac{h^{2}\left(G_{0}\right)}{8}\left(\frac{\rho}{g_{0}}+1\right) .
\end{aligned}
$$

Поскольку $h_{c}^{2}(R X) \geqslant h_{0} \cdot R X$ и $\lim _{X \rightarrow+\infty}\left(-h_{c}^{2}(R X)\right)=-\infty$, то $\exists X_{0}>s_{0}>0$ такое, что $\forall X>X_{0}$ выполнены оценки сверху

$$
A+\frac{1}{\alpha}\left(h_{c}^{2}\left(G_{0}\right)-h_{c}^{2}(R X)\right)<0
$$

И

$$
B+\frac{1}{\alpha}\left(h_{c}^{2}\left(G_{0}\right)-h_{c}^{2}(R X)\right)<0 .
$$

ТЕОРЕма 2.1 (об априорной оценке решения І-ой краевой задачи $(2.3)-(2.5)$ с условием сильного поглошения). Пусть функция (см. (1.6))

$$
v \in C(\bar{\omega}) \wedge C_{x, t}^{(2,1)}(\omega)
$$

является решением I-ой краевой задачи (2.3)-(2.5). Пусть существуют функция $g \in T_{h}(g)$, функции Тәклинда $h_{c}, h \in T(h)$, неубывающая функция $v_{0} \in$ $C[0,+\infty)$ такие, что для оператора Lv из (2.3) выполнено условие сильного поглощения:

$$
c(x, t)<h_{c}^{2}(G(|x|)) \quad \forall(x, t) \in \omega .
$$


Пусть (см. замечание 2.3) $r X>r X_{0}>s_{0}>0 u$

$$
\forall(x, t) \in \omega_{T} \cap\{G(|x|)<R X\} \equiv \omega_{T}(R)
$$

положено (см. (2.7))

$$
c_{0}(x, t) \stackrel{\text { def }}{=} c(x, t)-h_{c}^{2}(R X)<0
$$

и в случае (см. определение 2.3 и (2.1))

$$
(x, t) \in \omega_{T}(R) \cap\left\{|x| \geqslant s_{0}>1\right\}
$$

выполнены оченки сверху

$$
\begin{array}{cr}
0<2(3+q) \lambda_{1}(x, t) h^{2}(G(|x|)) \leqslant-g^{2}(|x|) c_{0}(x, t) & \text { npu } \quad n=1, \\
0<2 \lambda_{n}(x, t) \max \left[(3+q) h^{2}(G(|x|)), \frac{4(n-1)}{|x|} h(G(|x|)) g(|x|)\right] & n p u \quad n \geqslant 2, \\
\leqslant-g^{2}(|x|) c_{0}(x, t) &
\end{array}
$$

әде (см. (1.10), (1.11)) положено

$$
b(x, t)=\left(\sum_{i=1}^{n} b_{i}^{2}(x, t)\right)^{1 / 2}
$$

а в случае (см. (2.1) и замечание 2.3 )

$$
(x, t) \in \omega_{T}(R) \cap\left\{0<\rho \leqslant|x| \leqslant s_{0}\right\}
$$

выполнены оценки сверху

$$
\begin{aligned}
& 0<2(3+q) \lambda_{1}(x, t) \leqslant g_{0}^{2} \quad n p u \quad n=1, \\
& 0<2 \lambda_{n}(x, t) \max \left[(3+q) h\left(G_{0}\right), 4(n-1) / \rho\right] \leqslant g_{0}^{2} h\left(G_{0}\right) \quad \text { npu } \quad n \geqslant 2
\end{aligned}
$$

$u(c \mathcal{M} .(2.12))$

$$
b(x, t) \leqslant \frac{g_{0}}{8} h\left(G_{0}\right) .
$$

Пусть решение (2.6) I-ой краевой задачи (2.3)-(2.5) принадлежит классу OTT (2.2) и существуют постоянные $m_{H}^{\prime} \geqslant 0, m_{f} \geqslant 0 u m_{\varphi}^{\prime} \geqslant 0$ такие, что для функции $H$ из правой части уравнения (2.3), начальной функции $f$ из (2.4) 
и краевой функиии ч из (2.5), также взятых из класса ОТТ (2.2), выполнены либо оченки сверху:

$$
\begin{aligned}
H(x, t) \leqslant v_{0}(t) m_{H}^{\prime} & \forall(x, t) \in \omega, \\
f(x) \leqslant m_{f} & \forall(x, 0) \in \bar{\gamma}_{0}(\omega), \\
\varphi\left(M_{0}\right) \leqslant v_{0}\left(t_{0}\right) m_{\varphi}^{\prime} & \forall M_{0}=\left(x_{0}, t_{0}\right) \in \Sigma_{0}(\omega),
\end{aligned}
$$

либо оценки снизу:

$$
\begin{aligned}
& H(x, t) \geqslant-v_{0}(t) m_{H}^{\prime} \quad \forall(x, t) \in \omega, \\
& f(x) \geqslant-m_{f} \quad \forall(x, 0) \in \bar{\gamma}_{0}(\omega), \\
& \varphi\left(x_{0}, t_{0}\right) \geqslant-v_{0}\left(t_{0}\right) m_{\varphi}^{\prime} \quad \forall\left(x_{0}, t_{0}\right) \in \Sigma_{0}(\omega) .
\end{aligned}
$$

Тогда

$$
\forall(x, t) \in \bar{\omega}_{T} \cap\{G(|x|) \leqslant r X\}
$$

в классе ОТТ (2.2) при выполнении оценок сверху (2.16)-(2.18) имеют место оченки (при $0<r<1<4<R<+\infty)$

$$
\begin{aligned}
-v_{0}(t) \exp & \left\{\int_{0}^{G(|x|)} h(s) d s\right\} \leqslant v(x, t) \leqslant \exp \left\{h_{c}^{2}(R X) t\right\} \\
\times & {\left[m_{f}+v_{0}(T)\left(m_{H}^{\prime} t+m_{\varphi}^{\prime}+\exp \left\{-\frac{(1-r) R}{R-1} \int_{0}^{X} h(s) d s\right\}\right)\right], }
\end{aligned}
$$

и аналогично в классе ОТТ (2.2) при выполнении оценок снизу (2.19)-(2.21) имеют место оценки

$$
\begin{aligned}
v_{0}(t) \exp \{ & \left.\int_{0}^{G(|x|)} h(s) d s\right\} \geqslant v(x, t) \geqslant-\exp \left\{h_{c}^{2}(R X) t\right\} \\
& \times\left[m_{f}+v_{0}(T)\left(m_{H}^{\prime} t+m_{\varphi}^{\prime}+\exp \left\{-\frac{(1-r) R}{R-1} \int_{0}^{X} h(s) d s\right\}\right)\right] .
\end{aligned}
$$

ДокАЗАТЕЛЬСТво. Введем цилиндры (см. замечание 2.3)

$$
\amalg_{R X}(0, T]=\left\{(x, t) \in \mathbb{R}_{x, t}^{n+1} \mid G(|x|)<R X, 0<t \leqslant T\right\}
$$

и $\amalg_{r X}(0, T]$, где $0<r<1<4<R<+\infty$.

Положим

$$
\omega_{T}(R)=\omega_{T} \cap \amalg_{R X}(0, T], \quad \omega_{T}(r)=\omega_{T} \cap \amalg_{r X}(0, T] .
$$

Рассмотрим в $\omega_{T}(R) \subset \omega$, где область $\omega$ неограничена (по $x$ ) и удовлетворяет определению 2.1 с $y=0$, параболический оператор $L v$ из (1.10), (1.11), (2.3), для которого вьполнено условие сильного поглощения (2.7), так что

$$
0<c(x, t)<h_{c}^{2}(G(|x|))<h_{c}^{2}(R X) \quad \forall(x, t) \in \omega_{T}(R),
$$


и введем функцию (см. (2.6))

$$
w(x, t)=v(x, t) \exp \left\{-h_{c}^{2}(R X) t\right\}
$$

Тогда

$$
w \in C\left(\bar{\omega}_{T}(R)\right) \wedge C_{x, t}^{(2,1)}\left(\omega_{T}(R)\right),
$$

причем в силу (2.2) для функции $v$ из (2.6) имеем оценку ОТТ

$$
\begin{gathered}
|w(x, t)| \leqslant \exp \left\{-h_{c}^{2}(R X) t\right\}|v(x, t)| \leqslant v_{0}(T) \exp \left\{\int_{0}^{G(|x|)} h(s) d s\right\} \\
\forall(x, t) \in \bar{\omega}_{T}(R)
\end{gathered}
$$

и для функции (2.24).

Полагая (см. (1.10), (1.11) и (2.8))

$$
L_{0} \bar{w} \equiv \sum_{i, j=1}^{n} a_{i j}(x, t) D_{i} D_{j} \bar{w}+\sum_{i=1}^{n} b_{i}(x, t) D_{i} \bar{w}+c_{0}(x, t) \bar{w}-\frac{\partial \bar{w}}{\partial t} \quad \forall(x, t) \in \omega_{T}(R),
$$

видим, что функция $w$ из (2.24) является решением следующей I-ой краевой задачи:

$$
\begin{aligned}
L_{0} w(x, t) & \equiv \exp \left\{-h_{c}^{2}(R X) t\right\} L v(x, t)=-H_{0}(x, t) \\
& \equiv-H(x, t) \exp \left\{-h_{c}^{2}(R X) t\right\} \quad \forall(x, t) \in \omega_{T}(R),
\end{aligned}
$$

причем из $(2.4),(2.5)$ имеем

$$
w(x, 0)=v(x, 0)=f(x) \quad \forall(x, 0) \in \bar{\gamma}_{0}\left(\omega_{T}(R)\right)
$$

и

$$
w\left(M_{0}\right)=\varphi_{0}\left(M_{0}\right) \equiv \varphi\left(M_{0}\right) \exp \left\{-h_{c}^{2}(R X) t_{0}\right\} \quad \forall M_{0}=\left(x_{0}, t_{0}\right) \in \Sigma_{0} \cap \bar{\omega}_{T}(R) .
$$

В силу условий теоремы 2.1 для оператора $L_{0} w$ на множестве $\omega_{T}(R)$ выполнены условия $(2.10),(2.11)$ в случае $(2.9)$ и $(2.14),(2.15)$ в случае $(2.13)$, где $h \in T(h)$.

В цилиндре $\bar{Ц}_{R X}[0, T]$ при $\alpha=R /(R-1)>0$ введем барьер (не зависящий от $t$ ) при $h \in T(h), g \in T_{h}(g)$

$$
V(x)=v_{0}(T) \exp \left\{\alpha \int_{X}^{G(|x|)} h(s) d s\right\}
$$


ЛЕмма 2.1 (ср. [11]). Для функиий $V$ из (2.28) и ш из (2.24) справедливы утверждения:

1) $V \in C\left(\bar{\amalg}_{R X}[0, T]\right) \wedge C_{x, t}^{(2,1)}\left(\amalg_{R X}(0, T]\right)$;

2) $V(x) \geqslant v_{0}(T) \exp \left\{-\alpha \int_{0}^{X} h(s) d s\right\} \forall(x, t) \in \bar{\amalg}_{R X}[0, T]$;

3) $V(x)=v_{0}(T) \exp \left\{\alpha \int_{X}^{R X} h(s) d s\right\} \quad \forall(x, t) \in \Sigma\left(\bar{\amalg}_{R X}[0, T]\right) \equiv\{(x, t) \mid$ $G(|x|)=R X, \quad 0 \leqslant t \leqslant T\}$, причем

$$
V(x) \geqslant|w(x, t)| \quad \forall(x, t) \in \Sigma\left(\bar{\amalg}_{R X}[0, T]\right) \cap \bar{\omega}_{T}(R) ;
$$

4) $|w(x, t)| \leqslant v_{0}(T) \exp \left\{\int_{0}^{R X} h(s) d s\right\} \leqslant v_{0}(T) \exp \left\{\alpha \int_{X}^{R X} h(s) d s\right\} \forall(x, t) \in$ $\Sigma\left(\bar{\amalg}_{R X}[0, T]\right) \cap \bar{\omega}_{T}(R) ;$

5) $0<v_{0}(T) \exp \left\{-\alpha \int_{0}^{X} h(s) d s\right\} \leqslant V(x) \leqslant v_{0}(T) \exp \left\{-\alpha(1-r) \int_{0}^{X} h(s) d s\right\}$ $\forall(x, t) \in \overline{\mathrm{L}}_{r X}[0, T]$;

6) $L_{0} V(x)<0, \quad c_{0}(x, t)<0 \quad \forall(x, t) \in \omega_{T}(R) \equiv \omega_{T} \cap \amalg_{R X}(0, T]$.

ДокАЗАТЕльСтво. Утверждение 1) очевидно.

2) $G(|x|)>0 \forall|x|>\rho>0$, поэтому

$$
\alpha \int_{X}^{G(|x|)} h(s) d s=\alpha \int_{0}^{G(|x|)} h(s) d s-\alpha \int_{0}^{X} h(s) d s \geqslant-\alpha \int_{0}^{X} h(s) d s,
$$

что приводит к утверждению 2).

3) $\forall(x, t) \in \Sigma\left(\overline{\mathbf{L}}_{R X}[0, T]\right)$ имеем $G(|x|)=R X$ и при $\alpha=R /(R-1)$ получаем

$$
\frac{V(x)}{v_{0}(T)}=\exp \left\{\frac{R}{R-1} \int_{0}^{R X} h(s) d s\right\}=\exp \left\{\frac{R}{R-1}\left(\int_{0}^{R X} h(s) d s-\int_{0}^{X} h(s) d s\right\} .\right.
$$

Но $h \in T(h)$, так что $h(s) \uparrow$ на $[0,+\infty)$, и, учитывая, что $R>4>1>r>0$, имеем $h(s) \leqslant h(R s) \forall s>0$, что при замене $z=R s, d z=R d s$ дает оценку сверху

$$
\int_{0}^{X} h(s) d s \leqslant \int_{0}^{X} h(R s) d s=\frac{1}{R} \int_{0}^{R X} h(z) d z
$$

и тогда $\forall(x, t) \in \Sigma\left(\bar{\amalg}_{R X}[0, T]\right)$ имеем оценку снизу

$$
\frac{V(x)}{v_{0}(T)} \geqslant \exp \left\{\frac{R}{R-1}\left(1-\frac{1}{R}\right) \int_{0}^{R X} h(s) d s\right\}=\exp \left\{\int_{0}^{R X} h(s) d s\right\} \geqslant \frac{|w(x, t)|}{v_{0}(T)},
$$

так что и утверждение 3$)$ леммы доказано. Из утверждения 3$) \forall(x, t) \in \bar{\omega}_{T}(R) \cap$ $\Sigma\left(\bar{\amalg}_{R X}[0, T]\right)$ имеем оценку

$$
|w(x, t)| \leqslant v_{0}(T) \exp \left\{\int_{0}^{R X} h(s) d s\right\} \leqslant v_{0}(T) \exp \left\{\alpha \int_{X}^{R X} h(s) d s\right\},
$$


т.е. получено и утверждение 4) леммы.

Далее, $G(|x|) \leqslant r X \quad \forall(x, t) \in \bar{\amalg}_{r X}[0, T]$, что дает

$$
\begin{aligned}
\int_{X}^{G(|x|)} h(s) d s & =\int_{0}^{G(|x|)} h(s) d s-\int_{0}^{X} h(s) d s \leqslant \int_{0}^{r X} h(s) d s-\int_{0}^{X} h(s) d s \\
& =r \int_{0}^{X} h(r z) d z-\int_{0}^{X} h(s) d s<(r-1) \int_{0}^{X} h(s) d s
\end{aligned}
$$

(поскольку $0<r<1<4<R$, то $0<r s<s \forall s>0$, и, делая замену $s=r z$, $d s=r d z$, имеем $h(r z) \leqslant h(z) \forall z>0$ ), так что $\forall G(|x|) \leqslant r X$ имеем оценку

$$
\int_{X}^{G(|x|)} h(s) d s \leqslant(r-1) \int_{0}^{X} h(s) d s
$$

и тогда $\forall(x, t) \in \bar{\amalg}_{r X}[0, T]$ имеем из $(2.28)$

$$
V(x)<v_{0}(T) \exp \left\{-\alpha(1-r) \int_{0}^{X} h(s) d s\right\},
$$

т.е. утверждение 5) леммы доказано.

Для доказательства утверждения 6) леммы положим для краткости записи

$$
\begin{gathered}
g(|x|) \equiv g, \quad G(|x|) \equiv G, \quad h(G(|x|)) \equiv h(G), \quad D h(G(|x|)) \equiv D h(G) \\
a_{i j}(x, t) \equiv a_{i j}, \quad b_{i}(x, t) \equiv b_{i}, \quad i, j=\overline{1, n}, \quad c_{0}(x, t) \equiv c_{0}
\end{gathered}
$$

Тогда имеем $\forall|x| \geqslant \rho>0$

$$
\begin{aligned}
D_{i}|x| & =\frac{x_{i}}{|x|}, \quad|x| D_{i}|x|=x_{i}, \\
D_{i}\left(\frac{x_{i}}{|x|}\right) & =\frac{\delta_{i j}}{|x|}-\frac{x_{i} x_{j}}{|x|^{3}}, \\
D_{i} G & =\left\{\begin{array}{l}
\frac{x_{i}}{g_{0}|x|} \text { при } 0<\rho \leqslant|x| \leqslant 1, \\
\frac{1}{g} \frac{x_{i}}{|x|} \text { при } 1 \leqslant|x| \leqslant s_{0},
\end{array}\right. \\
D_{i} D_{j} G & =\left\{\begin{array}{l}
\frac{1}{g_{0}}\left(\frac{\delta_{i j}}{|x|}-\frac{x_{i} x_{j}}{|x|^{3}}\right), \\
-\frac{D_{g}}{g^{2}} \frac{x_{i} x_{j}}{|x|^{2}}+\frac{1}{g}\left(\frac{\delta_{i j}}{|x|}-\frac{x_{i} x_{j}}{|x|^{3}}\right), \quad 1 \leqslant|x| \leqslant s_{0},
\end{array}\right. \\
h(G) D_{i} G & =\left\{\begin{array}{l}
\frac{h_{0}}{g_{0}} \frac{x_{i}}{|x|}, \quad 0<\rho \leqslant|x| \leqslant 1, \\
\frac{h\left(|x| / g_{0}\right)}{g_{0}} \frac{x_{i}}{|x|}, \quad 1 \leqslant|x| \leqslant s_{0},
\end{array}\right.
\end{aligned}
$$




$$
\begin{aligned}
& h^{2}(G) D_{i} G D_{j} G= \begin{cases}h_{0}^{2} \frac{x_{i} x_{j}}{g_{0}^{2}|x|^{2}}, & 0<\rho \leqslant|x| \leqslant 1, \\
\frac{h\left(|x| / g_{0}\right)}{g_{0}^{2}|x|^{2}}, & 1 \leqslant|x| \leqslant s_{0},\end{cases} \\
& h(G) D_{i} D_{j} G= \begin{cases}\frac{h_{0}}{g_{0}}\left(\frac{\delta_{i j}}{|x|}-\frac{x_{i} x_{j}}{|x|^{3}}\right), & 0<\rho \leqslant|x| \leqslant 1, \\
h\left(\frac{|x|}{g_{0}}\right)\left(\frac{\delta_{i j}}{|x|}-\frac{x_{i} x_{j}}{|x|^{3}}\right), & 1 \leqslant|x| \leqslant s_{0},\end{cases} \\
& D h(G) D_{i} G D_{j} G= \begin{cases}0, & 0<\rho \leqslant|x| \leqslant 1, \\
\frac{D h\left(|x| / g_{0}\right) x_{i} x_{j}}{g_{0}^{2}|x|^{2}}, & 1 \leqslant|x| \leqslant s_{0},\end{cases}
\end{aligned}
$$

а также $\forall|x| \geqslant s_{0}>0$

$$
\begin{gathered}
D_{i} G=\frac{1}{g} \frac{x_{i}}{|x|}, \quad D_{i} D_{j} G=-\frac{D g}{g^{2}} \frac{x_{i} x_{j}}{|x|^{2}}+\frac{1}{g}\left(\frac{\delta_{i j}}{|x|}-\frac{x_{i} x_{j}}{|x|^{3}}\right) \\
h(G) D_{i} G=\frac{h(G)}{g} \frac{x_{i}}{|x|}, \quad h^{2}(G) D_{i} G D_{j} G=h^{2}(G) \frac{x_{i} x_{j}}{g^{2}|x|^{2}} \\
h(G) D_{i} D_{j} G=h(G)\left[-\frac{D g}{g^{2}} \frac{x_{i} x_{j}}{|x|^{2}}+\frac{1}{g}\left(\frac{\delta_{i j}}{|x|}-\frac{x_{i} x_{j}}{|x|^{3}}\right)\right] .
\end{gathered}
$$

И тогда $\forall|x| \geqslant \rho>0$ из (2.28) имеем

$$
\begin{gathered}
D_{i} V=\alpha V \frac{h(G)}{g} \frac{x_{i}}{|x|} \equiv \alpha V h(G) D_{i} G, \\
D_{i} D_{j} V=\alpha V\left[\left(\alpha h^{2}(G)+D h(G)\right) D_{i} G \cdot D_{j} G+h(G) D_{i} D_{j} G\right] \\
=\alpha V\left\{\frac{1}{g^{2}}\left(\alpha h^{2}(G)+D h(G)\right) \frac{x_{i} x_{j}}{|x|^{2}}\right. \\
\left.+h(G)\left[-\frac{D g}{g} \frac{x_{i} x_{j}}{|x|^{2}}+\frac{1}{g}\left(\frac{\delta_{i j}}{|x|}-\frac{x_{i} x_{j}}{|x|^{3}}\right)\right]\right\},
\end{gathered}
$$

и поэтому $\forall(x, t) \in \amalg_{R X}(0, T] \cap \omega_{T}(R)$ имеем $\forall|x| \geqslant \rho>0$ представление

$$
\frac{L_{0} V(x)}{\alpha V(x)}=\sum_{i=1}^{n} I_{i} \equiv J,
$$

где

$$
\begin{aligned}
& I_{1}=\frac{1}{g^{2}}\left[\alpha h^{2}(G)-D h(G)-h(G) D g\right] \sum_{i, j=1}^{n} a_{i j} \frac{x_{i} x_{j}}{|x|^{2}}, \\
& I_{2}=\frac{h(G)}{|x| g}\left[\sum_{i=1}^{n} a_{i i}-\sum_{i, j=1}^{n} a_{i j} \frac{x_{i} x_{j}}{|x|^{2}}\right], \\
& I_{3}=\frac{h(G)}{g} \sum_{i=1}^{n} b_{i} \frac{x_{i}}{|x|}, \quad I_{4}=\frac{c_{0}}{\alpha} .
\end{aligned}
$$


Учитывая, что $R>4, \alpha=R /(R-1)<4 / 3$, имеем

$$
\begin{gathered}
0<\alpha<2, \quad \frac{5}{8}<\frac{3}{4}<\frac{1}{\alpha}, \frac{\alpha+q+1}{2(q+3)}+\frac{1}{4}<\frac{3}{4}, \quad \frac{\alpha+q}{2(q+3)}+\frac{1}{4}<\frac{3}{4}, \\
\frac{\alpha+q+1}{2(q+3)}+\frac{1}{8}<\frac{1}{2}+\frac{1}{8}<\frac{3}{4} \Rightarrow \frac{\alpha+q+1}{2(q+3)}+\frac{1}{8}<\frac{3}{4} .
\end{gathered}
$$

Пусть сначала выполнено (2.9), тогда при $n=1$ имеем

$$
I_{2}=0, \quad a_{11}=\lambda_{1}, \quad b_{1}=b,
$$

и тогда из $(2.29)$ получаем

$$
J \leqslant \frac{\lambda_{1}}{g^{2}}\left[\alpha h^{2}(G)+D h(G)-h(G) D g\right]+h(G) \frac{b}{g}+\frac{c_{0}}{\alpha} .
$$

Но в силу определения 2.3

$$
D h(G) \leqslant q h^{2}(G), \quad-D g \leqslant h(G),
$$

т.е. при $n=1$

$$
J \leqslant \lambda_{1} h^{2}(G) \frac{\alpha+q+1}{g^{2}}+b \frac{h(G)}{g}+\frac{c_{0}}{\alpha} .
$$

Но в силу (2.9)-(2.11) при $n=1$

$$
2(3+q) \lambda_{1} h^{2}(G) \leqslant-g^{2} c_{0}, \quad b h(G) \leqslant-\frac{g c_{0}}{8},
$$

так что, учитывая (2.8), имеем из (2.30) оценку сверху:

$$
J \leqslant\left[\alpha\left(\frac{\alpha+q+1}{2(3+q)}+\frac{1}{8}\right)\right] c_{0} \leqslant\left(\frac{1}{5}-\frac{5}{8}\right) c_{0}<0,
$$

т.е. в случае $(2.9)$ при $n=1$ имеем $L_{0} V(x)<0$. При $n \geqslant 2$ в случае $(2.9)$ имеем

$$
0<\lambda_{1} \leqslant \sum_{i, j=1}^{n} a_{i j} \frac{x_{i} x_{j}}{|x|^{2}} \leqslant \lambda_{n}
$$

откуда

$$
0<\sum_{i=1}^{n} a_{i i}-\sum_{i, j=1}^{n} a_{i j} \frac{x_{i} x_{j}}{|x|^{2}} \leqslant(n-1) \lambda_{n} .
$$

Вновь учитывая оценки $D h(G) \leqslant q h^{2}(G),-D g \leqslant h(G)$ из определения 2.3 и оценки (см. (2.11))

$$
\left|\sum_{i=1}^{n} \frac{b_{i} x_{i}}{|x|}\right| \leqslant b,
$$


в случае $(2.9)$ при $n \geqslant 2$ имеем

$$
\begin{aligned}
J & \leqslant \frac{\lambda_{n}}{g^{2}}\left[\alpha h^{2}(G)+D h(G)-h(G) D g\right]+\frac{(n-1) \lambda_{n} h(G)}{|x| g}+b \frac{h(G)}{g}+\frac{c_{0}}{\alpha} \\
& \leqslant \frac{\lambda_{n}}{g^{2}} h^{2}(G)(\alpha+q+1)+\frac{n-1}{|x| g} h(G)+\frac{G h(G)}{g}+\frac{c_{0}}{\alpha}
\end{aligned}
$$

откуда, учитывая, что при $n \geqslant 2$ в силу (2.9)-(2.11) имеем

$$
2 \lambda_{n} \max \left[(3+q) h^{2}(G), \frac{4(n-1) h(G) g}{|x|}\right] \leqslant-g^{2} c_{0}, \quad 8 b h(G) \leqslant-g c_{0},
$$

так что

$$
\lambda_{n} \frac{h^{2}(G)}{g^{2}} \leqslant-\frac{c_{0}}{2(3+q)}, \quad \frac{(n-1) \lambda_{n} h(G)}{|x| g} \leqslant-\frac{c_{0}}{8}, \quad \frac{b h(G)}{g} \leqslant-\frac{c_{0}}{8} .
$$

Но тогда при $n \geqslant 2$ в случае $(2.9)$ в силу (2.8) имеем из $(2.30)$

$$
J \leqslant c_{0}\left[\frac{1}{\alpha}-\left(\frac{\alpha+q+1}{2(3+q)}+\frac{1}{2}\right)\right] \leqslant c_{0}\left(\frac{1}{\alpha}-\frac{3}{4}\right)<0,
$$

так что в случае (2.9) оценка $L_{0} V(x)<0$ доказана и при $n \geqslant 2$.

В случае $(2.13)$ в силу $(2.14),(2.15)$ и (2.8) имеем при $n=1$, используя определение 2.3 , оценку

$$
J \leqslant \frac{\lambda_{1}}{g_{0}^{2}}\left(\alpha h^{2}\left(G_{0}\right)+d_{0}\right)+\frac{h\left(G_{0}\right)}{g_{0}} b+\frac{c_{0}}{\alpha} h^{2}\left(G_{0}\right) \leqslant A+\frac{c_{0}}{\alpha},
$$

где

$$
A=\frac{\alpha h^{2}\left(G_{0}\right)+d_{0}}{2(3+q)}+\frac{g_{0}}{8} h^{2}\left(G_{0}\right) .
$$

Далее, в случае (2.13) при $n \geqslant 2$ имеем оценку

$$
J \leqslant \lambda_{n}\left[\alpha h^{2}\left(G_{0}\right)+d_{0}\right]+\frac{(n-1)}{g_{0}} h\left(G_{0}\right) \lambda_{n}+\frac{h\left(G_{0}\right) b}{g_{0}}+\frac{c_{0}}{\alpha} \leqslant B+\frac{c_{0}}{\alpha}
$$

где

$$
B=\frac{\alpha h^{2}\left(G_{0}\right)+d_{0}}{2(3+q)}+\frac{h^{2}\left(G_{0}\right)}{8}\left(1+\frac{\rho}{g_{0}}\right) .
$$

Но поскольку в силу (2.8)

$$
c_{0} \leqslant h_{c}^{2}\left(G_{0}\right)-h_{c}^{2}(R X)<0,
$$

то в случае (2.13) получаем оценки

$$
J \leqslant A+\frac{1}{\alpha}\left(h_{c}^{2}\left(G_{0}\right)-h_{c}^{2}(R X)\right) \text { при } n=1
$$


и

$$
J \leqslant B+\frac{1}{\alpha}\left(h_{c}^{2}\left(G_{0}\right)-h_{c}^{2}(R X)\right) \text { при } n \geqslant 2 .
$$

Но тогда из замечания 2.3 , считая, что $X>X_{0}>s_{0}$ в $\amalg_{R X}(0, T]$, получаем оценку сверху $J<0$ в случае (2.13) как при $n=1$, так и при $n \geqslant 2$. Утверждение 6 ) леммы доказано.

Рассмотрим теперь І-ю краевую задачу (2.25)-(2.27) для функции $(2.24)$, считая выполненными в теореме 2.1 оценки сверху (2.16)-(2.18). Тогда из (2.25)-(2.27) имеем оценки сверху:

$$
\begin{aligned}
H_{0}(x, t) & \leqslant H(x, t) \leqslant v_{0}(T) m_{H}^{\prime} \quad \forall(x, t) \in \omega_{T}(R), \\
f(x) & \leqslant m_{f} \quad \forall(x, 0) \in \bar{\gamma}_{0}\left(\omega_{T}(R)\right), \\
\varphi_{0}\left(M_{0}\right) & \equiv \varphi\left(M_{0}\right) \exp \left\{-h_{c}^{2}(R X) t_{0}\right\} \\
& \leqslant v_{0}(T) m_{\varphi}^{\prime} \quad \forall M_{0}=\left(x_{0}, t_{0}\right) \in \Sigma_{0}(\omega) \cap \bar{\amalg}_{R X}[0, T] .
\end{aligned}
$$

Введем барьер (см. (2.28))

$$
W(x, t)=V(x)-w(x, t)+m_{\varphi_{0}}(T)+m_{f}+v_{0}(T) m_{H_{0}}^{\prime} \cdot t .
$$

Тогда в силу леммы 2.1

$$
L_{0} V(x)<0 \quad \forall(x, t) \in \omega_{T}(R),
$$

и в силу (2.31)

$$
-L_{0} w(x, t) \leqslant v_{0}(T) m_{H}^{\prime} \quad \forall(x, t) \in \omega_{T}(R),
$$

и в силу (2.8)

$$
c_{0}(x, t)\left(m_{\varphi_{0}}(T)+m_{f}\right) \leqslant 0, \quad-v_{0}(T) m_{H}^{\prime} \leqslant 0,
$$

т.e.

$$
L_{0} W(x, t)<0, \quad c_{0}(x, t)<0 \quad \forall(x, t) \in \omega_{T}(R),
$$

так что функция $W$ суперпараболична на множестве $\omega_{T}(R)$. Далее, $\forall(x, 0) \in$ $\bar{\gamma}_{0}\left(\omega_{T}(R)\right)$ имеем в силу $(2.26),(2.32)$

$$
W(x, 0)=V(x)-w(x, 0)+m_{\varphi_{0}}(T)+m_{f},
$$

где $V(x)>0$ в силу утверждения 2) леммы 2.1 и $-w(x, 0)=-v(x, 0)=-f(x)$, так что (см. $(2.32))$

$$
W(x, 0)>m_{f}-f(x) \geqslant 0 \quad \forall(x, 0) \in \bar{\gamma}_{0}\left(\omega_{T}(R)\right)
$$

Далее,

$$
\forall M_{0}=\left(x_{0}, t_{0}\right) \in \Sigma_{0}(\omega) \cap \bar{\amalg}_{R X}[0, T]
$$


имеем

$$
W\left(x_{0}, t_{0}\right)=V\left(x_{0}\right)-\varphi_{0}\left(x_{0}, t_{0}\right)+m_{\varphi_{0}}(T)+v_{0}(T) m_{H_{0}}^{\prime} t_{0} .
$$

В силу $(2.33) \varphi_{0}\left(x_{0}, t_{0}\right) \leqslant m_{\varphi}(T)$. Но из утверждения 2$)$ леммы $V\left(x_{0}\right)>0$, так что при выполнении (2.33) имеем (см. (2.34))

$$
W\left(M_{0}\right) \geqslant m_{\varphi_{0}}(T)-\varphi_{0}\left(M_{0}\right)+m_{f}+v_{0}(T) m_{H}^{\prime} t_{0}>0 .
$$

Далее, отметим представление

$$
\Sigma\left(\omega_{T}(R)\right)=\left(\Sigma(\omega) \cap \bar{\amalg}_{R X}[0, T]\right) \cup\left(\Sigma\left(\bar{\amalg}_{R X}[0, T]\right) \cap \bar{\omega}_{T}\right) .
$$

Тогда в силу утверждения 4) леммы 2.1 имеем

$$
\begin{aligned}
W\left(M_{0}\right) & \geqslant V\left(x_{0}\right)-\left|w\left(M_{0}\right)\right|+m_{\varphi_{0}}(T)+v_{0}(T) m_{H}^{\prime} t_{0} \\
& \geqslant V\left(x_{0}\right)-\left|w\left(x_{0}, t_{0}\right)\right|>0 \quad \forall M_{0} \in \Sigma\left(\bar{\amalg}_{R X}[0, T]\right) \cap \bar{\omega}_{T},
\end{aligned}
$$

откуда получаем оценку снизу:

$$
W\left(M_{0}\right)>0 \quad \forall M_{0} \in \Sigma\left(\bar{\amalg}_{R X}[0, T]\right) \cap \bar{\omega}_{T},
$$

так что

$$
\begin{gathered}
W\left(M_{0}\right) \geqslant 0 \\
\forall M_{0} \in \Sigma_{0}\left(\omega_{T}(R)\right) \equiv\left(\Sigma_{0}(\omega) \cap \bar{\amalg}_{R X}[0, T]\right) \cup\left(\Sigma\left(\bar{\amalg}_{R X}[0, T]\right) \cap \bar{\omega}_{T}(R)\right) .
\end{gathered}
$$

Но тем самым доказана оценка снизу:

$$
W(x, t) \geqslant 0 \quad \forall(x, t) \in \Gamma\left(\omega_{T}(R)\right)=\bar{\gamma}_{0}\left(\omega_{T}(R)\right) \cup \Sigma_{0}\left(\omega_{T}(R)\right) .
$$

В силу слабого принщипа минимума (см. теорему 1.2), примененного к суперпараболической (на множестве $\omega_{T}(R)$ ) функции $W$, имеем оценку снизу:

$$
W(x, t) \geqslant 0 \quad \forall(x, t) \in \bar{\omega}_{T}(R),
$$

из которой, в частности, получаем оценку сверху для решения I-ой краевой задачи $(2.25)-(2.27) w(x, t)$ из $(2.24)$ :

$$
w(x, t) \leqslant V(x)+m_{\varphi_{0}}(T)+m_{f}+v_{0}(T) m_{H_{0}}^{\prime} t \quad \forall(x, t) \in \bar{\omega}_{T}(R),
$$

из которой в силу утверждения 5) леммы 2.1 имеем оценку сверху:

$$
\begin{aligned}
& w(x, t) \leqslant v_{0}(T) m_{\varphi_{0}}^{\prime}+m_{f}+v_{0}(T) m_{H_{0}}^{\prime} t \\
&+v_{0}(T) \exp \left\{-\alpha(1-r) \int_{0}^{X} h(s) d s\right\} \\
& \forall(x, t) \in \bar{\omega}_{T}(r) \subset \bar{\amalg}_{r X}[0, T] .
\end{aligned}
$$

Но тогда, учитьвая оценку ОТТ (2.2), из (2.24) получаем оценки (2.22) теоремы 2.1 при выполнении оценок сверху (2.16)-(2.18). 
СлЕДСТвИЕ 2.1 (теорема единственности в классе ОТТ решения I-ой краевой задачи для оператора $L v$ с условием сильного поглощения). Пусть функция (2.6) является решением I-ой краевой задачи (2.3)-(2.5) при выполнении условия сильного поглощения (2.7), взятое из класса ОТТ (2.2). Пусть в оценках (2.16)-(2.21) положено $m_{H}^{\prime}=m_{f}=m_{\varphi}^{\prime}=0$. Тогда для любой фиксированной точки $(x, t) \in \bar{\omega}_{T}$ существует множество

$$
\bar{\omega}_{T}(r)=\bar{\omega}_{T} \cap \bar{\amalg}_{r X}[0, T]
$$

$\left(\right.$ при $X>X_{0}>s_{0}$ из замечания 2.3), содержащее точку $(x, t)$, и в силу двусторонней оценки (см. (2.22), (2.23)) теоремы 2.1 имеем

$$
|w(x, t)| \leqslant v_{0}(T) \exp \left\{-\alpha(1-r) \int_{0}^{X} h(s) d s\right\} \quad \forall(x, t) \in \bar{\omega}_{T}(r) .
$$

Переходя в ней к пределу при $X \rightarrow+\infty$, получаем $w(x, t)=0 \forall(x, t) \in \bar{\omega}_{T}$, т.е. доказано, что $w(x, t) \equiv 0$ в $\bar{\omega}_{T}$, откуда, поскольку (см. $\left.(2.24)\right) \forall(x, t) \in \bar{\omega}_{T}(R)$

$$
v(x, t)=\exp \left\{h_{c}^{2}(R X) t\right\} w(x, t),
$$

имеем $v(x, t) \equiv 0$ в $\bar{\omega}_{T}$.

\section{Список литературы}

1. Камынин Л.И., Химченко Б.Н. О слабом (алгебраическом) принципе экстремума для параболической системы 2-го порядка // Изв. РАН. Сер. матем. 1997. Т. 61. № 5. C. $35-62$.

2. Камынин Л. И., Химченко Б. Н. О слабом (стохастическом) принципе экстремума для одного класса параболических систем 2-го порядка // Вестн. РУДН. 1996. Т. 3. № 1. C. $58-71$.

3. Камынин Л. И., Химченко Б. Н. Об изотропной теории единственности решения задачи Коши для параболического уравнения второго порядка // Дифференц. уравн. 1988. T. 24. № 1. С. $73-85$.

4. Камынин Л.И., Химченко Б.Н. О проблеме Тихонова-Петровского для параболических уравнений 2-го порядка // Сиб. матем. журн. 1981. Т. 22. № 5. С. 78-109.

5. Хорн Р., Джонсон Ч. Матричный анализ. М.: Мир, 1989.

6. Ильин A.M., Калашников A.C., Олейник О. А. Линейные уравнения второго порядка параболического типа // УМН. 1961. Т. 17. № 3. С. 3-146.

7. Ладыженская О. А., Солонников В. А., Уральцева Н. Н. Линейные и квазилинейные уравнения параболического типа. М.: Наука, 1967.

8. Фридман A. Уравнения с частными производными параболического типа. М.: Мир, 1968.

9. Тихонов A. Н., Самарский A. А. Уравнения математической физики. М.: Наука, 1972.

10. Камынин Л.И., Химченко Б.Н. Об односторонних оценках решений задачи Коши для параболических уравнений второго порядка в классах быстро растущих функций. I // Дифференц. уравн. 1994. Т. 30. №5. С. 838-846.

11. Камынин Л. И. Односторонние оценки решения І краевой задачи в неограниченной области для сильно диссипативного параболического уравнения 2-го порядка // Сиб. матем. журн. 1994. Т. 35. № 1. С. 105-117. 